\title{
Backaction Effects of a SSET Measuring a Qubit Spectroscopy and Ground State Measurement
}

\author{
Benjamin Turek, Johannes Majer, Aashish Clerk, Steve Girvin, Andreas Wallraff, Kevin Bladh, David Gunnarsson, \\ Tim Duty, Per Delsing, and Robert Schoelkopf
}

\begin{abstract}
We investigate the backaction of superconducting single-electron transistor (SSET) continuously measuring a Cooper-pair box. Due to the minimized backaction of the SSET, we observe a $2 e$ periodic Coulomb staircase according to the twolevel system Hamiltonian of the Cooper-pair box. We demonstrate that we can control the quantum broadening of the ground state in-situ. We perform spectroscopy measurements and demonstrate that we have full control over the Cooper-pair box Hamiltonian. The ability to reduce the backaction is a necessary condition to use the SSET as a quantum state readout for the CPB as a qubit.
\end{abstract}

Index Terms-Quantum computing, superconducting devices.

\section{INTRODUCTION}

A $\mathrm{N}$ interesting question in solid-state quantum computation is how to measure a qubit and how the measurement process influences the qubit. Recently there has been considerable experimental progress using superconducting circuits to realize the qubit and the meter that measures the qubit. Many of the these devices are based on the single-Cooper-pair box [1], [2]. Coherent oscillations in such a Cooper-pair box have been observed [3]-[5] as well as Rabi oscillations [6] and Ramsey oscillation [7]. Despite these encouraging results, the measuring device and the influence of the measurement on the qubit are not yet completely understood.

In this article we report measurements where the Cooper-pair box is measured using a superconducting single-electron transistor (SSET). The SSET can be operated such that it continuously and weakly measures the charge of the Cooper-pair box. However, it also couples noise to the Cooper-pair box. This effect is called backaction and influences the states of the box in different ways. One can divide this influence in four categories, in order of decreasing severity, as follows:

First, the SSET can create nonequilibrium quasiparticles in the box. Therefore the states of the box are not described by Cooper-pair tunneling alone, and the box is no longer a simple two-level system. Quasiparticle poisoning is often [5], [8], [9], but not always [2], [4] observed in SSET measurements of the

Manuscript received October 4, 2004. This work was supported in part by the National Security Agency and Advanced Research and Development Activity under the Army Research Office, the NSF, the David and Lucile Packard Foundation, and the W. M. Keck Foundation.

B. Turek, J. Majer, A. Clerk, S. Girvin, A. Wallraff, and R. Schoelkopf are with the Department of Applied Physics and Physics, Yale University, New Haven, CT 06511 USA (e-mail: johannes.majer@yale.edu).

K. Bladh, D. Gunnarsson, T. Duty and P. Delsing are with the Microtechnology Center at Chalmers MC2, Department of Microelectronics and Nanoscience, Chalmers University of Technology and Göteborg University, SE-412 96, Göteborg, Sweden.

Digital Object Identifier 10.1109/TASC.2005.850096 box, but its origin is not well understood. Second, even if there are no quasiparticles present, the SSET can excite the qubit. Now the box is still described by a two-level Hamiltonian, however the system does not stay in the ground state but is in a mixture of ground and excited state. Third, SSET's backaction can cause increased relaxation. After the box has been brought to the first excited state, the noise of the SSET can destroy the state of the box by extracting energy and bringing the system back to the ground state. The fourth category is the dephasing caused by the measurement process [10]. By the fundamental laws of quantum mechanics, measuring a system perturbs its state, and specifically destroys the phase of a superposition. Therefore this form of backaction is the fundamental limit.

The purpose of this article is to investigate the first two manifestations of backaction and to demonstrate that we were able to reduce them to observe the box in the $2 e$-periodic ground state. Furthermore, we show that the box obeys a simple spin-1/2 Hamiltonian in which both terms can be controlled in-situ. We perform continuous-wave spectroscopy on the box, measuring the energy level separation and the avoided crossing of the charge states. We find that the quantum broadening of the Coulomb staircase is consistent with the level repulsion observed in spectroscopy.

\section{THE COOPER-PAIR BOX}

The Cooper-pair box consists of a superconducting island which is connected to a superconducting lead via a Josephson junction (Fig. 1). Another gate lead allows one to change the electrostatic potential of the island with the application of a voltage $V_{g b}$ through the capacitance $C_{g b}$. The state of the island is described by the number of Cooper-pairs on the island. Because the Josephson energy $E_{J}$ is smaller than four times the charging energy $E_{c}=e^{2} / 2 C_{\Sigma}$, one has to consider only two charging states. The Cooper-pair box is described by the following Hamiltonian

$$
\hat{H}=\frac{E_{e l}}{2} \hat{\sigma}_{z}+\frac{E_{J}}{2} \hat{\sigma}_{x} \quad E_{e l}=4 E_{c}\left(n_{g b}-1\right)
$$

where $\sigma_{z}$ and $\sigma_{x}$ are the Pauli matrices. $n_{g b}$ is the number of electrons induced by the gate electrode $n_{g b}=C_{g b} V_{g b} / e$. The Josephson junction consists of two junctions in parallel, forming a SQUID loop [2] (see Fig. 1). The effective Josephson coupling $E_{J}$ of these two junctions can be tuned with the magnetic flux $\Phi$ through the loop: $E_{J}=E_{J}^{\max }\left|\cos \left(\pi \Phi / \Phi_{0}\right)\right|$. Here $\Phi_{0}=$ $h / 2 e$ is the superconducting flux quantum. Therefore the split Cooper-pair box is described by the two-level Hamiltonian (1), where both terms can be controlled during the experiment. 

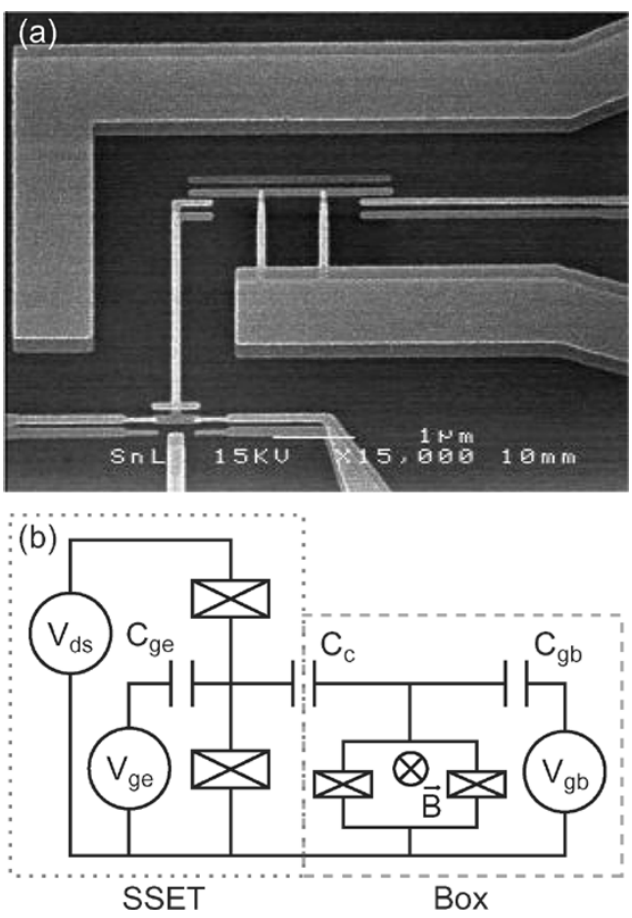

Fig. 1. (a) A scanning electron micrograph (SEM) of the device. The device is fabricated using shadow evaporation technique [11]. (b) The circuit diagram for the Cooper-pair box coupled with $C_{C}$ to the superconducting-single-electron transistor.

The states and the energy levels of the system can by found by diagonalizing the Hamiltonian (1). The energy difference between the ground state and the excited state is given by

$$
\Delta E=\sqrt{E_{J}^{2}+\left(4 E_{c}\right)^{2}\left(n_{g b}-1\right)^{2}}
$$

Far away from the degeneracy point $\left(\left|n_{g b}-1\right| \gg 0\right)$ the eigenstates are given by pure charge states. However in the vicinity of the degeneracy point $\left(\left|n_{g b}-1\right| \approx E_{J} / 4 E_{c}\right)$ the eigenstates are superpositions of charge states and the energy levels show an avoided crossing.

The charge of the box in the ground state is given by the expectation value of the charge operator $\hat{q}=e\left(1+\hat{\sigma}_{z}\right)$ in the ground state:

$$
\langle q\rangle_{\substack{\text { sround } \\ \text { state }}}=e\left(1+\frac{n_{g b}-1}{\sqrt{\left(\frac{E_{J}}{4 E_{c}}\right)^{2}+\left(n_{g b}-1\right)^{2}}}\right)
$$

Without any Josephson coupling $\left(E_{J}=0\right)$ the box charge versus applied gate voltage (i.e. Coulomb staircase) is just a simple step function, which is 0 for $n_{g b}<1$ and 2 for $n_{g b}>1$. However with a finite Josephson energy $E_{J}$, a superposition of charge states exists in the region where $E_{J} \approx E_{e l}$ and therefore the step becomes broadened. The larger the value of $E_{J}$ gets, the broader the step function. The charge of the box in the excited state is given by $\langle q\rangle_{\text {excited state }}=2 e-\langle q\rangle_{\text {ground state }}$.

\section{MEASUREMENTS}

The sample was measured in a dilution refrigerator at $13 \mathrm{mK}$. This thermal energy is far less than the relevant energy scales

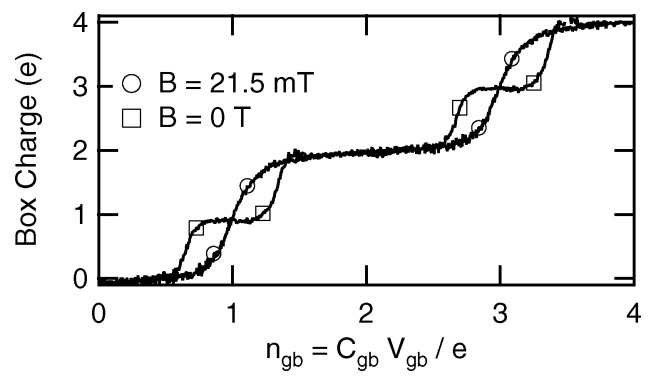

Fig. 2. The Coulomb staircase, i.e. box charge versus induced gate charge $n_{g b}$, without (square symbols) and with (circular symbols) magnetic field applied. The magnetic field affects the superconducting gap and removes the quasiparticle poisoning.

of either the SSET or the box. The charge of the box is measured with a SSET placed nearby (Fig. 1). Via the capacitor $C_{C}$, a small fraction $\left(C_{C} / C_{\Sigma}=2.5 \%\right)$ of the charge of the box island couples to the SSET island. The SSET is operated as an radio-frequency single-electron transistor (RF-SET) [12], which is an outstanding electrometer. The sensitivity of the electrometer as well as the backaction noise [13] depend strongly on the operation point of the SSET, which can be tuned with the drain-source voltage $V_{D S}$ and the SSET gate voltage $V_{g e}$.

First we biased the SSET on the gap rise. The measured box charge as a function of the applied gate voltage $V_{g b}$, is completely $1 e$ periodic, similar to the observations by Männik $e t$ al. [9]. At this bias point $\left(V_{d s}=4 \Delta / e \approx 1 \mathrm{mV}\right)$, the current through the SSET breaks many pairs in the drain and source lead of the SSET, though the power dissipated is only $1-10 \mathrm{pW}$. This apparently induces nonequilibrium quasiparticles in the box, though there is no direct connection between them and the mechanism is not known. This backaction noise is of the first kind as described above.

We then bias the SSET on the double Josephson quasiparticle process (DJQP) [13], [14], which occurs at lower SSET drain-source voltage. Fig. 2 shows with square symbols the measured Coulomb staircase. The Coulomb staircase is $2 e$-periodic, however at odd number of electrons an intermediate step occurs due to quasiparticle poisoning. However after applying a magnetic field of $20 \mathrm{mT}$ perpendicular to the substrate, the small step disappears (Fig. 2 circular symbols). The Coulomb staircase follows exactly the theoretical prediction (3). Applying a magnetic field lowers the superconducting gap in the aluminum, and could reduce the gap in the larger leads more than in the thin island [15]. The quasiparticle states in the leads would have a lower energy than on the island and therefore the quasiparticles can not tunnel on the island and poison the Coulomb staircase, as observed in SSET's [16]. A similar method has been used by Duty et al. [4] where a large magnetic field parallel to the device is applied. In contrast to previous experiments by Lehnert et al. [8] the box measured here has a smaller charging energy and therefore the quasiparticle states are more separated. In conclusion, using the optimal bias point of the SSET, applying a magnetic field and reducing the charging energy of the box allows us to measure a full Coulomb staircase that is not quasiparticle poisoned. Hence, we can avoid the backaction of the first category.

We measured the Coulomb staircase as function of the applied magnetic field (Fig. 3). One observes that the Coulomb 


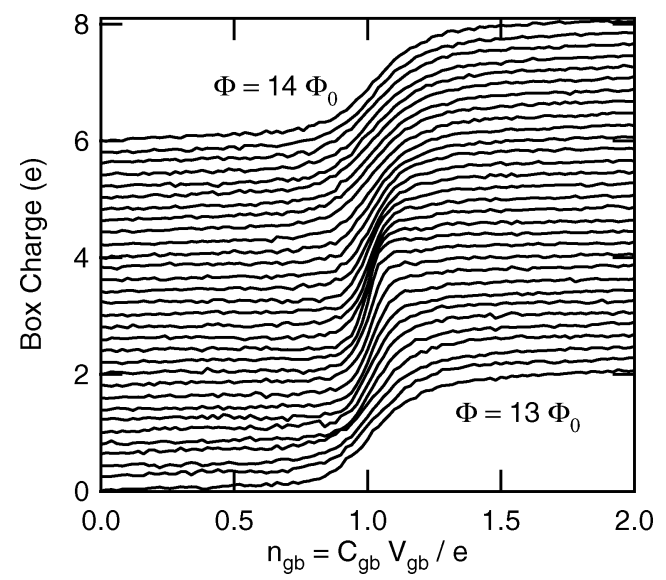

Fig. 3. Coulomb staircase for different magnetic field values. The staircase is periodically broadened and sharpened as a function of the applied flux. For integer flux quanta, i.e. large $E_{J}$, the staircase is maximally broadened and for half integer flux quanta, i.e. small $E_{J}$, step-like. Note: $13 \Phi_{0}$ correspond to 27 $\mathrm{mT}$.

staircase periodically sharpens and broadens. The period is consistent with the number of flux quanta in the split box junction. At integer flux quanta the Josephson energy is maximal and the Coulomb staircase is maximally broadened. At half integer flux, the Josephson energy is suppressed and the Coulomb staircase approaches a step-like function. We fit the theoretical expression (3) to the staircases, which allows us to extract the energy ratio between Josephson and charging energy $\left(E_{J} / 4 E_{c}\right)$. This ratio as a function of magnetic field is plotted in Fig. 5.

The shape of the Coulomb staircase in Fig. 3 is not generic. In order to observe these curves the SSET has to be biased slightly below the DJQP resonance. At this bias point theory [13] predicts that the backaction noise is primarily relaxing the box, i.e. there is backaction noise of the third category, but no backaction of the second kind. The box is therefore forced into the ground state by the noise of the SSET.

To observe the excited state and the energy spectrum of the box, we perform spectroscopy by applying a continuous microwave signal to the gate of the Cooper-pair box. When the microwave energy $h \nu$ (where $h$ is Planck's constant) matches the energy difference between the ground and excited state, the microwaves induce a transition from the ground state to the excited state. The system can be put in a mixture of ground and excited state at two discrete points in gate charge where the excitation is resonant. This microwave response appears as an extra peak and dip in the Coulomb staircase. We measured the Coulomb staircase with and without microwave signal and subtracted them to separate out the microwave induced response. Fig. 4(a) shows the microwave peak and dip as a function of the applied microwave frequency. The position of peak and dip follow the expected hyperbolic behavior with an avoided crossing of about $15 \mathrm{GHz}$. By fitting the positions with the expression for the energy level difference (2) we can extract the Josephson energy $E_{J}=14.9 \mathrm{GHz}$ and the charging energy $E_{c}=18.9 \mathrm{GHz}$. This measurement was performed at integer flux quanta applied and therefore maximal $E_{J}$. One observes that the peak and dip height disappear toward the degeneracy point. This is due to the fact that the eigenstates are superposition of charge states and
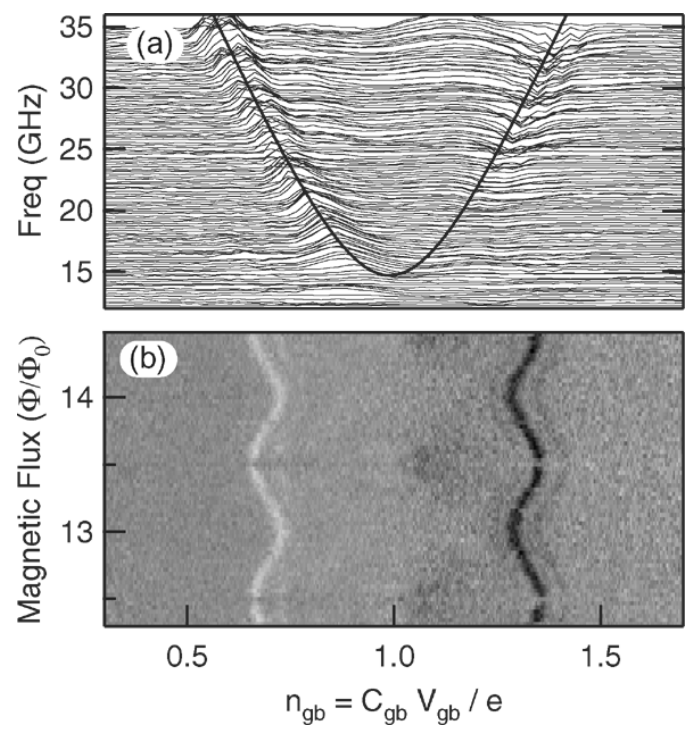

Fig. 4. Microwave spectroscopy results. Obtained by measuring the Coulomb staircase with microwaves applied and subtracting the staircase without microwaves. (a) Spectroscopy versus microwave frequency. From the fit with the hyperbola (Eq. (2)) we obtain $E_{J}=14.9 \mathrm{GHz}$ and $E_{c}=18.9 \mathrm{GHz}$. (b) Spectroscopy versus magnetic field. A microwave signal with a frequency of $25 \mathrm{GHz}$ is applied. The peak and dip oscillate as a function of the applied flux. For integer flux quanta, i.e. large $E_{J}$, the avoided energy level crossing is large and the resonances move inwards, toward the degeneracy point $\left(n_{g b}=1\right)$. Note: each plot is 10 hours of data without any offset charge jump.

the difference of the box charge between the ground state and the excited state becomes small and disappears at the degeneracy point. The fact that our Coulomb staircase is not quasiparticle poisoned is very important, because it allows us to observe the spectroscopy signal down to the degeneracy point.

We also performed spectroscopy for a constant frequency of $25 \mathrm{GHz}$ and varying magnetic fields (Fig. 4(b)). One observes that the peak and dip positions oscillate periodically with the applied the flux. As the $E_{J}$ becomes larger, the avoided crossing is larger and therefore the energy levels are more rounded. Hence the position, where the microwave frequency is in resonance with the energy level difference, moves toward the degeneracy point $\left(n_{g b}=1\right)$. One can also observe that the signal disappears at the positions where $E_{J}$ is minimal (i.e. half integer flux quanta). When $E_{J}$ is zero, the eigenstates are pure charge states. Our microwave excitation is applied to the gate and is therefore a charge excitation. Since only a perpendicular component can induce transitions between states, the microwave signal is not able to drive the transition.

The spectroscopically obtained values of $E_{J}$ and $E_{c}$ versus magnetic field are shown in Fig. 5 (empty symbols) and can be compared with the values derived from the ground state. One observes that the two curves, obtained in completely different measurements, agree very well. This confirms that the measured Coulomb staircase is indeed the ground state of the two-level system and that the broadening is only due to quantum fluctuations. Hence we demonstrate that the Cooper-pair box is not affected by backaction of the second category. One observes a small discrepancy of the two measurements at low values of $E_{J}$. This is possibly due to $1 / f$ charge noise which additionally broadens the step. 


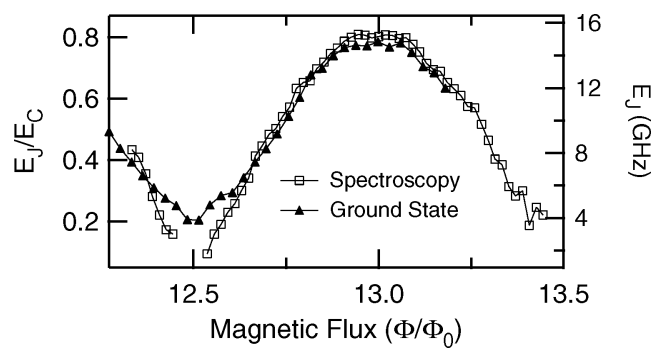

Fig. 5. Energy ratio $E_{J} / E_{c}$ versus magnetic field, obtained from the broadening of the ground state (solid symbols) and from the spectroscopy (open symbols).

\section{CONCLUSION}

In these experiments we demonstrate that we are able to eliminate the two most severe forms of backaction on the Cooper-pair box: The backaction that creates nonequilibrium quasiparticles on the Cooper-pair box and the backaction noise that excites the box from the ground to the excited state. With biasing the SSET at the optimal position and applying a small field, we are able to observe the ground state of the Cooper-pair box without quasiparticle poisoning. Spectroscopy and ground state measurements demonstrate that the Cooper-pair box is behaving according to the simple two-level Hamiltonian (1) and that we are able to control both terms in-situ. The good agreement between spectroscopy and ground state results shows that we are indeed observing the ground state of the system.

However, as discussed above, the SSET may still be relaxing the box, i.e. backaction of the third category. Further measurements will address the backaction induced contribution to the relaxation.

\section{ACKNOWLEDGMENT}

The authors would like to thank M. H. Devoret, D. Esteve, K. Lehnert, L. Frunzio, and J. Teufel for useful discussions.

\section{REFERENCES}

[1] M. Büttiker, "Zero-current potential drop across small-capacitance Josephson junctions," Phys. Rev. B, vol. 36, pp. 3548-3555, 1987.

[2] V. Bouchiat, D. Vion, P. Joyez, D. Esteve, and M. H. Devoret, "Quantum coherence with a single Cooper pair,” Phys. Scr., vol. T76, pp. 165-170, 1998.

[3] Y. Nakamura, Y. A. Pashkin, and J. S. Tsai, "Coherent control of macroscopic quantum states in a single-Cooper-pair box," Nature, vol. 398, pp. 786-788, 1999.

[4] T. Duty, D. Gunnarsson, K. Bladh, and P. Delsing, "Coherent dynamics of a Josephson charge qubit," Phys. Rev. B, vol. 69, p. 140 503(R), 2004.

[5] A. Guillaume, J. F. Schneiderman, P. Delsing, H. M. Bozler, and P. M. Echternach, "Free evolution of superposition states in a single Cooper pair box," Phys. Rev. B, vol. 69, p. 132 504, 2004.

[6] Y. Nakamura, Y. A. Pashkin, and J. S. Tsai, "Rabi oscillations in a Josephson-junction charge two-level system," Phys. Rev. Lett., vol. 87 , p. $246601,2001$.

[7] D. Vion, A. Aassime, A. Cottet, P. Joyez, H. Pothier, C. Urbina, D. Esteve, and M. H. Devoret, "Manipulating the quantum state of an electrical circuit," Science, vol. 296, pp. 886-889, 2002.

[8] K. W. Lehnert, K. Bladh, L. F. Spietz, D. Gunnarsson, D. I. Schuster, P. Delsing, and R. J. Schoelkopf, "Measurement of the excited-state lifetime of a microelectronic circuit," Phys. Rev. Lett., vol. 90, p. 027002 , 2003.

[9] J. Männik and J. E. Lukens, "Effect of measurement on the periodicity of the Colomb staircase of a superconducting box," Phys. Rev. Lett., vol. 92, p. $057004,2004$.

[10] M. H. Devoret and R. J. Schoelkopf, "Amplifying quantum signals with the single-electron transistor," Nature, vol. 406, pp. 1039-1046, 2000.

[11] G. J. Dolan, "Offset masks for lift-off photoprocessing," Appl. Phys. Lett., vol. 31, p. 337, 1977.

[12] R. J. Schoelkopf, P. Wahlgren, A. A. Koshevnikov, P. Delsing, and D. E. Prober, "The radio-frequency single-electron transistor (RF-SET): a fast and ultrasensitive electrometer," Science, vol. 280, pp. 1238-1242, 1998.

[13] A. A. Clerk, S. M. Girvin, A. K. Nguyen, and A. D. Stone, "Resonant Cooper-pair tunneling: quantum noise and measurement characteristics," Phys. Rev. Lett., vol. 89, p. 176 804, 2002.

[14] S. Pohlen, "The Superconducting Single Electron Transistor," Ph.D. thesis, Harvard, 1999.

[15] A. Anthore, H. Pothier, and D. Esteve, "Density of states in a superconductor carrying a supercurrent," Phys. Rev. Lett., vol. 90, p. 127 001, 2003.

[16] J. Aumentado, M. W. Keller, J. M. Martinis, and M. H. Devoret, "Nonequilibrium quasiparticles and 2e periodicity in single-Cooper-pair transistors," Phys. Rev. Lett., vol. 92, p. 066 802, 2004. 